\title{
Analysis of Quality Control Connecting Rod with Statistical Process Control (SPC) Method in PT.XYZ
}

\author{
Nanang Supriana ${ }^{1}$, Muhammad Ali Akbar ${ }^{2}$, Osep Hijuzaman \\ Industrial Engineering of STT. Wastukancana Purwakarta, West Java, Indonesia
}

\begin{abstract}
Quality controlof a product is very important to do that levels approaching zero defect generated. PT.XYZ produce connecting rod with a data defect during January 2015 - March 2015 as much as $7.47 \%$ of the total production of 26070 pcs. The statistical methods used for data processing using methods of Statistical Process Control (SPC) or Control Chart, Process Capability and Fishbone Diagram. The decline in the number of visible defect after the repair to $3.65 \%$ of the total production of 27053 pcs and the value of Process Capability $(\mathrm{CP})$ rose from 0.26 to 1.27.
\end{abstract}

Keyword: Quality, Defect, Capability Process , Statistical ProcessControl(SPC) Method

\section{Introduction}

Quality control aimed at improving the quality of the product, should ideally be carried out at each stage of the process development and manufacture of such products. Thus the quality control should have started from the design, receipt of raw materials to the manufacturing process. To produce a good product as desired then quality control has been an absolute requirement. Quality control is important to be done by the company to the products produced in accordance with standards established by the company as well as standards set by local agencies and international managing of quality standardization / quality, and of course in accordance with what is expected by the consumer

PT. XYZ as automotive companies that have international scale can not be liberated and be separated from the already very long journey for the existence of four-wheeled vehicles for this type of bus and truck in Indonesia. With the growing level of competition will require good quality control so that it can compete with products produced by other companies. Currently the products are of high quality is the key factor of success of competition.

\section{Literatur Review}

\subsection{Definition of Quality}

"Quality is very important for a product, either in the form of goods and services. Things that are very important for manufacturers with regard to the products are: quality, cost and productivity. The quality is the ability of a product or service that consistently meets the expectations of consumers. Thus the quality is the only thing that matters most to both pihak.Dalam many cases, the concept of quality is different between manufacturers / producers and customers / consumers "(Rev. Dorothea, 1999).

Definition of Statistical Quality Control "Statistical Process Control (SPC) is a process used to monitor the standards, make measurements and take corrective action while a product or service being produced" (Heizer and Barry Render, 2005.)

"Statistical quality control is a problem-solving techniques that are used to monitor, control, analyze, manage, and improve products and processes using statistical methods. Statistical quality control (statistical quality control) is often referred to as statistical process control (statistical process control). Statistical quality control and statistical process control is the two terms are interchangeable, which, if done together then the user will see the picture of the performance of the process of the present and the future. "(Feigenbaum, Armand V, 1991)

"Statistical quality control using SPC (Statistical Process Control) has seven (7) major statistical tools that can be used as tools for quality control, among others, namely; checksheet, histogram, control chart, Pareto diagram, cause and effect diagrams, scatter diagrams and process "(Garvin, David, 2001)

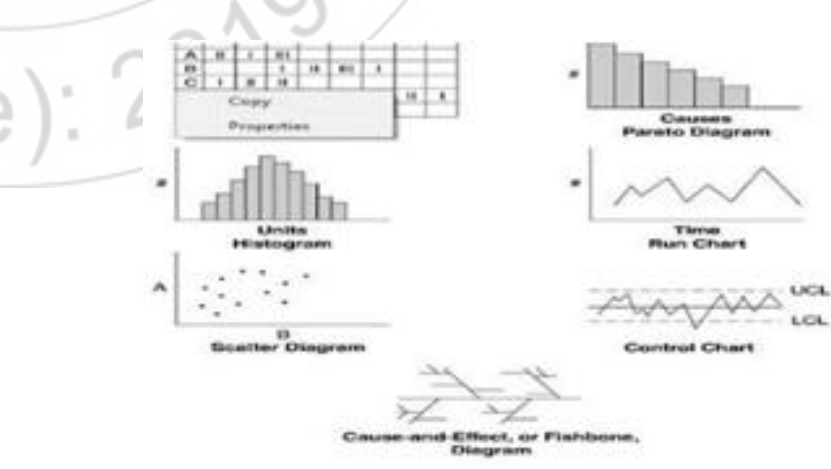

Figure 1: QC Seven Tool

\subsection{Control Chart}

"Control chart is a statistical method to distinguish the variation or deviation due to general causes and causes khusus.Peta describe improved quality control, consisting of two situations, the first is when the map control is made, the 


\section{International Journal of Science and Research (IJSR) \\ ISSN (Online): 2319-7064}

Index Copernicus Value (2013): 6.14 | Impact Factor (2015): 6.391

process in unstable conditions. Conditions beyond the control limit occurs due to special causes (assignable cause), then look for corrective action so that the process becomes stable. The result is an improvement process. The control chart can be divided into two groups according to the type of data, namely a control chart for attribute data and control chart for variable data ". (Dorothea Revelation, 1999)

\subsection{P Control chart}

In analyzing the data of this study, use $\mathrm{p}$ control chart (control chart proportion of damage) as a tool for statistical process control. The use of $\mathrm{p}$ control map are due to the quality control is performed attributes, as well as data obtained as sample observation is not fixed and the product which has such damage can not be repaired anymore and should be rejected (reject).

\subsection{CapabilityProcess (Cp)}

Process capability is the concept of process capability put forward in the early 1970s with the introduction "Capability Ratio" by Juran, a renowned authority on quality. After nearly 40 years on, many indexes such as Cp, Cpk and Cpm developed for the measurement process. Capability means the ability of a process of producing a product that meets specifications. If the process has a good capability, the process will generate located within the limits of the specification limits. Conversely if the process has the capability ugly, the process will produce who is beyond specification limits sehinggamenimbulkan losses because many products that will be rejected. Process capability index $(\mathrm{Cp})$ is calculated using the following formula:

$$
C p=\frac{U S L-L S L}{6 \sigma}
$$

where :

$\mathrm{Cp}=$ Capability Process

USL = Upper Standard Limit

LSL = Lower Standard Limit

$6 \sigma \quad=6$ Deviation

\section{Methodology}

The research methodology is the steps and plans of the thinking process in solving the problem, ranging from preliminary research, the discovery of the problem, observation, data collection both written and reference directly into the field of observation, data processing until the conclusion on the issues examined. 


\section{International Journal of Science and Research (IJSR) \\ ISSN (Online): 2319-7064}

Index Copernicus Value (2013): 6.14 | Impact Factor (2015): 6.391

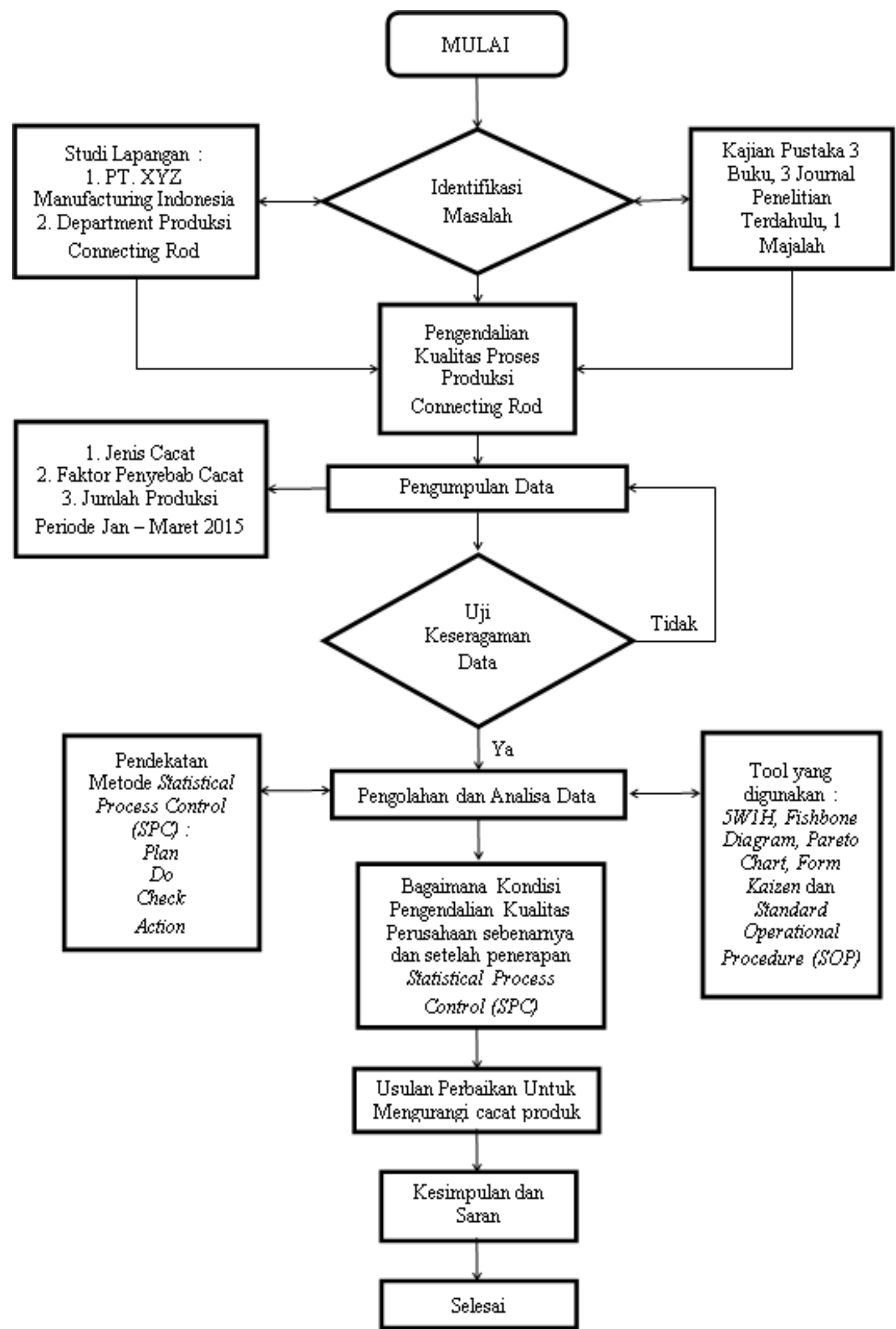

Figure 2: Framework Troubleshooting

\section{Discussion and Analysis}

In the face of increasingly fierce competition, the company is expected to produce high quality products, in accordance with the standards set by the company and consumer demand. Therefore, the company must carry out quality control activities continuously to the product. The control carried out by the company, namely: Control of raw materials and the control of production processes, from the research, there are several much damage that occurs in product yield Connecting Rod, and is still quite high even exceeding the tolerance limit damage to the product specified by the company. Such damage can be complex or simple nature. The company should strive to be able to resolve problems that arise immediately. The types of defects that occur on the Connecting Rod, among others: Diameter Oval, not processed, rust, scratches and chipped. 


\section{International Journal of Science and Research (IJSR) \\ ISSN (Online): 2319-7064}

Index Copernicus Value (2013): 6.14 | Impact Factor (2015): 6.391

Table 1: Number and type of disability

\begin{tabular}{|c|c|c|c|c|}
\hline Defect Condition & $\begin{array}{c}\text { Amount } \\
\text { Defect }\end{array}$ & $\begin{array}{c}\% \\
\text { Defect }\end{array}$ & $\begin{array}{c}\text { Akumulasi } \\
\text { Defect }\end{array}$ & $\begin{array}{c}\% \text { Akumulasi } \\
\text { Defect }\end{array}$ \\
\hline Diameter Oval & 1949 & $50 \%$ & 1949 & $50 \%$ \\
\hline Not processed & 1109 & $28 \%$ & 3058 & $78 \%$ \\
\hline Rust & 355 & $9 \%$ & 3413 & $87 \%$ \\
\hline Scratch & 347 & $9 \%$ & 3760 & $96 \%$ \\
\hline Chipped & 177 & $4 \%$ & 3937 & $100 \%$ \\
\hline Amount & 3937 & $100 \%$ & \multicolumn{3}{|l}{} \\
\cline { 1 - 2 } &
\end{tabular}

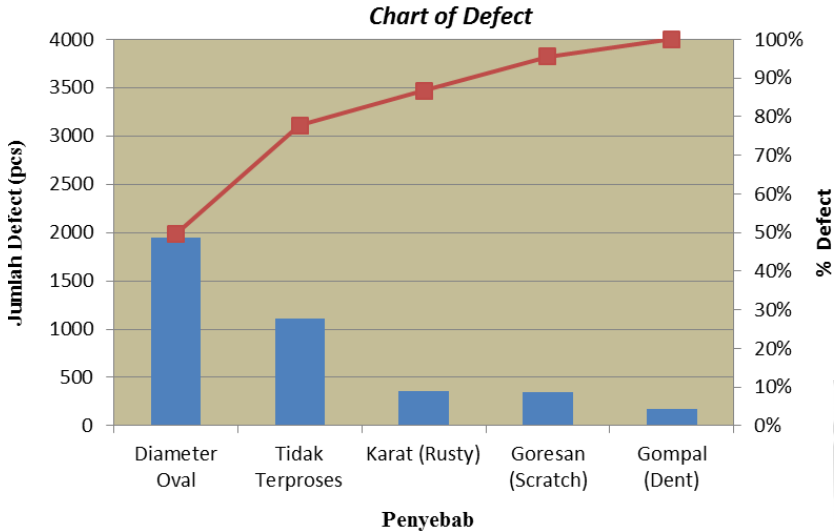

Figure 3: Condition Causes Defect Pareto January 2015-March 2015

Uji Kecukupan Data

Perhitungan uji kecukupan data dilakukan dengan menggunakan tingkat kepercayaan dipilih $95 \%$ dan tingkat ketelitian $5 \%$, dengan rumus sebagai berikut :

$\mathrm{k}=99 \%=3$

$\mathrm{s} \quad=1 \%=0.01$

$\mathrm{k} / \mathrm{s} \quad=3 / 0.01=300$

$\mathrm{N} \quad=21$

Kriteria yang digunakan adalah apabila sampel yang sudah digunakan (N) lebih besar atau sama dengan jumlah sampel yang seharusnya $\left(N^{\prime}\right)$, maka data atau sampel yang digunakan sudah mencukupi. Namun apabila jumlah sampel yang sudah digunakan $(\mathrm{N})$ lebih kecil atau sama dengan jumlah sampel yang seharusnya ( $\left.N^{\prime}\right)$, maka sampel atau data yang telah diambil tidak mencukupi, Berdasarkan data yang ada maka perhitungannya adalah :

$N^{\prime}=\left[\frac{k / s \sqrt{N \sum x^{2}-\left(\sum x\right)^{2}}}{\sum x}\right]^{2}$

$N^{t}=\left[\frac{40 \sqrt{21 * 32537748-(26070)^{2}}}{26070}\right]^{2}$

$N^{\prime}=\left[\frac{76396.94}{26070}\right]^{2}$

$N^{\prime}=8.58=9$

Based on these calculations, it was found that the value $\mathrm{N}$ 'is smaller than the value of $\mathrm{N}$ is $9<21$, meaning that the data or samples collected are sufficient.

\section{Control Chart}

The steps to create a control chart:

a. Calculating the center line / Central Line (CL)Line center / Central Line is the middle line that was ushered upper control limit (UCL) and lower control limit (LCL). The center line is the line that represents the average level of damage in a production process. To calculate the center lines use the formula:

$$
C L=\bar{p}=\frac{\sum n p}{\sum n}
$$

Remark :

$\Sigma \mathrm{np}=$ Amount of Defect

$\Sigma \mathrm{n}=$ Amount of Checked

Based on the above formula, found the Central Line (CL) as follows:

$\Sigma \mathrm{np}=3937$

$\Sigma \mathrm{n}=26070$

$$
C L=\bar{p}=\frac{\sum 3937}{\sum 26070}=0.151
$$

b. Calculating upper control limit Upper Control Limit (UCL)

To calculate the upper control limit (Upper Control Limit / UCL) conducted by the formula:

$$
U C L=\bar{p}+3\left(\sqrt{\frac{\bar{p}(1-\bar{p})}{n}}\right.
$$

Remark :

Subgrup 1- subgrup 4

$\bar{p}=$ Average of defect

$n=$ Amount of production

and etc...

c. Calculating the lower control limit or Lower Control Limit (LCL)

To calculate the lower control limit or LCL performed by the formula::

$$
\begin{aligned}
& \mathrm{UCL}=\bar{p}+2\left(\sqrt{\frac{\bar{p}(1-\bar{p})}{\bar{n}}}\right)=0.075+2\left(\sqrt{\left.\frac{0.078(0.922)}{1308}\right)}=0.090\right. \\
& \mathrm{UCL}=\bar{p}+2\left(\sqrt{\left.\frac{\bar{p}(1-\bar{p})}{\bar{n}}\right)}=0.075+2\left(\sqrt{\left.\frac{0.078(0.922)}{1284}\right)}=0.090\right.\right. \\
& \mathrm{UCL}=\bar{p}+2\left(\sqrt{\frac{\bar{p}(1-\bar{p})}{\bar{n}}}\right)=0.075+2\left(\sqrt{\left.\frac{0.078(0.922)}{1372}\right)}=0.090\right. \\
& \mathrm{UCL}=\bar{p}+2\left(\sqrt{\left.\frac{\bar{p}(1-\bar{p})}{\bar{n}}\right)}=0.075+2\left(\sqrt{\left.\frac{0.078(0.922)}{1376}\right)}=0.090\right.\right. \\
& \mathrm{LCL}=\bar{p}-2\left(\sqrt{\left.\frac{\bar{p}(1-\bar{p})}{\bar{n}}\right)}\right.
\end{aligned}
$$

Remark :

Subgrup 1- subgrup 4

$\bar{p}=$ Average of defect

$n=$ Amount of production

and etc...

note: If the LCL $<0$ then LCL considered $=0$

Then the calculation is as follows: 


\section{International Journal of Science and Research (IJSR) \\ ISSN (Online): 2319-7064}

Index Copernicus Value (2013): 6.14 | Impact Factor (2015): 6.391

$\mathrm{LCL}=\bar{p}-2\left(\sqrt{\frac{\bar{p}(1-\bar{p})}{\bar{n}}}\right)=0.075-2\left(\sqrt{\left.\frac{0.078(0.922)}{1308}\right)}=0.060\right.$

$\mathrm{LCL}=\bar{p}-2\left(\sqrt{\left.\frac{\bar{p}(1-\bar{p})}{\bar{n}}\right)}=0.075-2\left(\sqrt{\left.\frac{0.078(0.922)}{1284}\right)}=0.060\right.\right.$

$\mathrm{LCL}=\bar{p}-2\left(\sqrt{\left.\frac{\bar{p}(1-\bar{p})}{\bar{n}}\right)}=0.075-2\left(\sqrt{\left.\frac{0.078(0.922)}{1372}\right)}=0.060\right.\right.$

$\mathrm{LCL}=\bar{p}-2\left(\sqrt{\frac{\bar{p}(1-\bar{p})}{\bar{n}}}\right)=0.075-2\left(\sqrt{\left.\frac{0.078(0.922)}{1376}\right)}=0.060\right.$

and etc...

For the calculation of $\mathrm{p}$ control map which can be seen in the following table:

Table 2: Calculation Period Month Limit Full Jan- March 2015

\begin{tabular}{|c|c|c|c|c|c|c|}
\hline $\begin{array}{l}\text { Tanggal } \\
\text { Produksi } \\
\text { (Jan- } \\
\text { Mar'15) }\end{array}$ & $\begin{array}{l}\text { Jumlah } \\
\text { Produksi } \\
\text { (pcs) }\end{array}$ & $\begin{array}{c}\text { Jumlah } \\
\text { Produk } \\
\text { Rusak } \\
\text { (Defect) } \\
\text { (pcs) }\end{array}$ & $\begin{array}{c}\text { Present ase } \\
\text { Produk } \\
\text { Rusak } \\
\text { (Defect) }\end{array}$ & CL & UCL & LCL \\
\hline 1 & 1308 & 177 & 0.135 & 0.075 & 0.090 & 0.060 \\
\hline 2 & 1284 & 140 & 0.109 & 0.075 & 0.090 & 0.060 \\
\hline 3 & 1372 & 152 & 0.111 & 0.075 & 0.090 & 0.060 \\
\hline 4 & 1376 & 178 & 0.129 & 0.075 & 0.090 & 0.060 \\
\hline 5 & 1040 & 165 & 0.159 & 0.075 & 0.090 & 0.060 \\
\hline 6 & 1356 & 152 & 0.112 & 0.075 & 0.090 & 0.060 \\
\hline 7 & 1308 & 162 & 0.124 & 0.075 & 0.090 & 0.060 \\
\hline 8 & 1176 & 176 & 0.150 & 0.075 & 0.090 & 0.060 \\
\hline 9 & 1288 & 156 & 0.121 & 0.075 & 0.090 & 0.060 \\
\hline 10 & 1252 & 238 & 0.190 & 0.075 & 0.090 & 0.060 \\
\hline 11 & 1228 & 202 & 0.164 & 0.075 & 0.090 & 0.060 \\
\hline 12 & 1260 & 163 & 0.129 & 0.075 & 0.090 & 0.060 \\
\hline 13 & 1188 & 218 & 0.184 & 0.075 & 0.090 & 0.060 \\
\hline 14 & 1000 & 216 & 0.216 & 0.075 & 0.090 & 0.060 \\
\hline 15 & 1192 & 227 & 0.190 & 0.075 & 0.090 & 0.060 \\
\hline 16 & 1192 & 190 & 0.159 & 0.075 & 0.090 & 0.060 \\
\hline 17 & 1228 & 214 & 0.174 & 0.075 & 0.090 & 0.060 \\
\hline 18 & 1250 & 181 & 0.145 & 0.075 & 0.090 & 0.060 \\
\hline 19 & 1252 & 218 & 0.174 & 0.075 & 0.090 & 0.060 \\
\hline 20 & 1260 & 174 & 0.138 & 0.075 & 0.090 & 0.060 \\
\hline 21 & 1260 & 238 & 0.189 & 0.075 & 0.090 & 0.060 \\
\hline TOTAL & 26070 & 3937 & 3.203 & & & \\
\hline $\begin{array}{l}\text { RATA- } \\
\text { RATA }\end{array}$ & 1241.43 & 189.71 & & & & \\
\hline
\end{tabular}

From the calculation table 2 above, it can then be made $p$ control map which can be seen in the image below:

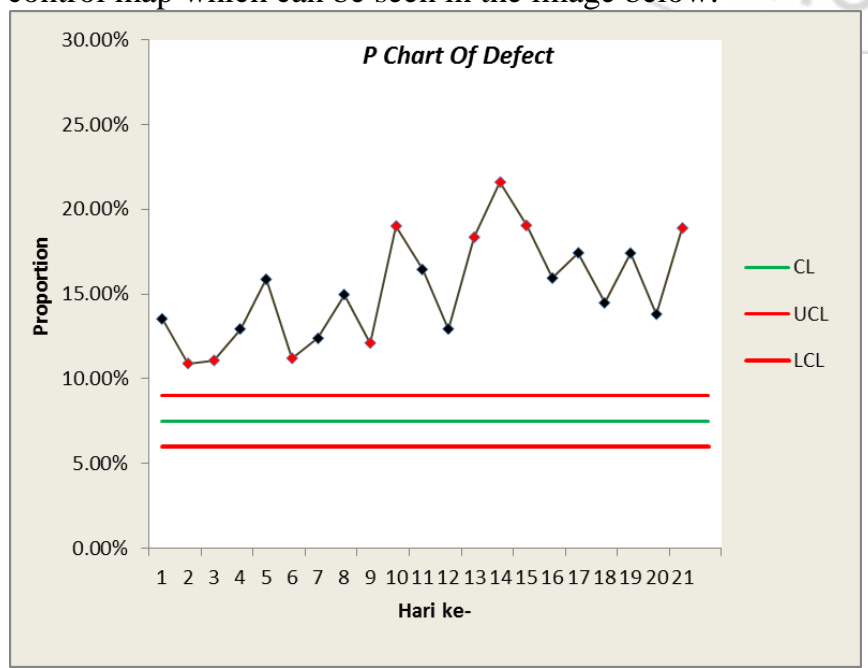

Figure 4: Map Control Defect proportion of the period Jan March 2015
Based on the above map image $p$ control can be seen that the data obtained entirely outside the control limit that has been set even, so it can be said that the process is out of control.

\section{Analysis of Capability Process}

Activities that include statistical technique that is used all round the production process that includes development prior to production, in order to quantify process variability, variability analysis relative to the requirements or specifications of the product that is useful for the development and production in eliminating or reducing the amount of variability is called process capability analysis.

Table 3: Data Inspection Connecting Rod Diameter Hole Model W04D Month January 2015 - March 2015 (Before Repair)

\begin{tabular}{|c|c|c|c|}
\hline \multirow{2}{*}{$\begin{array}{c}\text { Tanggal } \\
\text { Produksi } \\
\text { (diurutkan) }\end{array}$} & \multicolumn{2}{|r|}{ Diameter Hole } & Maret 2015 \\
\hline & \multicolumn{3}{|c|}{ Stan dard $666 \quad 0 / 0.015$} \\
\hline $\mathbf{1}$ & -0.005 & -0002 & -0.008 \\
\hline $\mathbf{2}$ & -0.000 & -0005 & -0.0003 \\
\hline $\mathbf{3}$ & -0.006 & -0009 & -0.008 \\
\hline 4 & -0.000 & -0001 & -0.014 \\
\hline 5 & -0.000 & -0005 & -0.002 \\
\hline 6 & -0.005 & -0006 & -0.011 \\
\hline $\mathbf{7}$ & $-0.00 \mathrm{~s}$ & $-000 \%$ & -0.008 \\
\hline $\mathbf{8}$ & -0.012 & $-\mathbf{0 0 0 8}$ & -0.011 \\
\hline 9 & -0.009 & -0010 & -0.012 \\
\hline $\mathbf{v}$ & $-0.0 \mathrm{II}$ & $-\mathbf{0 0 n}$ & -0.010 \\
\hline $\mathbf{n}$ & $-0.08 \mathrm{~B}$ & -0009 & -0.007 \\
\hline $\mathbf{z}$ & -0.014 & $-000 s$ & $\mathbf{0 0 0 2}$ \\
\hline $\mathbf{B}$ & $-0.0 \mathrm{H}$ & -0008 & -0.018 \\
\hline $\mathbf{T}$ & -0.00 & -0003 & -0.004 \\
\hline $\mathbf{E}$ & -0.009 & -0005 & -0.013 \\
\hline $\mathbf{w}$ & -0.008 & $-\mathbf{0 0 0 s}$ & -0.015 \\
\hline $\mathbf{U}$ & -0.009 & -0007 & -0008 \\
\hline $\mathbf{E}$ & -0.098 & -0005 & -0.005 \\
\hline $\mathbf{Q}$ & $-0.0 \mathrm{~s}$ & -0003 & -0.003 \\
\hline $\mathbf{2 0}$ & $-0.0 \mathrm{II}$ & -0005 & -0.005 \\
\hline $\mathbf{z}$ & $-0.00 \mathrm{TS}$ & -0009 & -0.007 \\
\hline
\end{tabular}

Based on the above data it can be seen:

1. Capability Process $(\mathrm{CP})$ Connecting RodonJanuary 2015prior to the change as follows:
USL $=0$

LSL $\quad=-0.015$

With an average of Data $=-0.017$

Standar Deviation $(\sigma)=0.024$

a. Process Capability Ratio or Capability Process Index / $\mathrm{Cp}$

$$
\begin{aligned}
C p & =\frac{\text { USL - LSL }}{6 \sigma} \\
& =\frac{0-(-0.015)}{6.0,024} \\
& =0.102
\end{aligned}
$$

So, $\mathrm{Cp}<1$, process uncapable 


\section{International Journal of Science and Research (IJSR) \\ ISSN (Online): 2319-7064}

Index Copernicus Value (2013): 6.14 | Impact Factor (2015): 6.391

b. Upper and lower capability index

$$
\begin{aligned}
& \mathrm{CPU}=\frac{\mathrm{USL}-\mathrm{U}_{-}}{3 \sigma}=\frac{0-(-0.017)}{3 \cdot 0.024}=0.233 \\
& \mathrm{CPL}=\frac{\mathrm{LSL}}{3 \sigma}=\frac{0.017-(-0.015)}{3 \cdot 0.024}=-0.029
\end{aligned}
$$

c. Cpk index

$$
\begin{aligned}
\mathrm{Cpk} & =\min \left(\frac{\mathrm{USL}-\mu, \mu}{3 \sigma}, \frac{\mu-\mathrm{LSL}}{3 \sigma}\right) \\
& =\operatorname{Min}(\mathrm{Cpu}, \mathrm{Cpl}) \\
& =\operatorname{Min}(0.233,-0.029) \\
& =-0.029
\end{aligned}
$$

Cpk $\leq 1$, process uncapable

Whereas if Minitab software are as follows : CP Diameter Hole Bulan Januari 2015

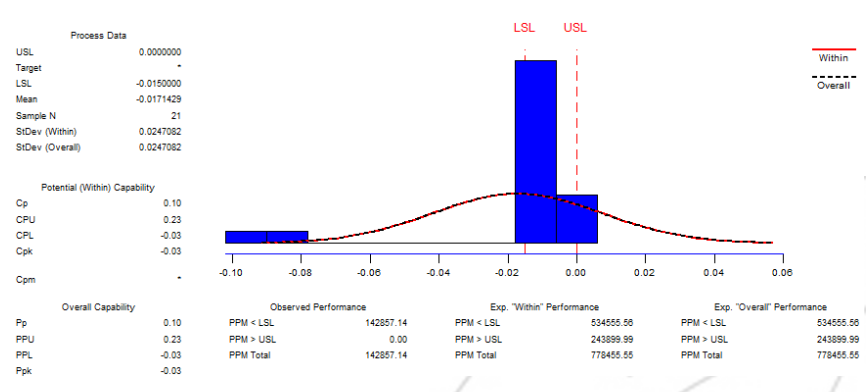

2. Capability Process (CP)Connecting RodonFebruari 2015prior to the change as follows:

$$
\begin{array}{ll}
\text { USL } & =0 \\
\text { LSL } & =-0.015
\end{array}
$$

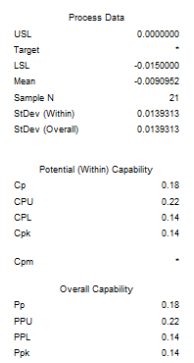

CP Diameter Hole Bulan Februari 2015

a. Process Capability Ratio or Capability Process Index / Cp

$$
\begin{aligned}
\mathrm{Cp}_{\mathrm{p}} & =\frac{\text { USL }- \text { LSL }}{6 \sigma} \\
& =\frac{0-(-0.015)}{6.0 .005} \\
& =0.502
\end{aligned}
$$

So $\mathrm{Cp}<1$, process uncapable

b. Upper and lower capability index

$$
\begin{aligned}
& \mathrm{CPU}=\frac{\mathrm{USL}-\mu}{3 \sigma}=\frac{0-(-0.007)}{3 \cdot 0.005}=0.485 \\
& \mathrm{CPL}=\frac{\mu-\mathrm{LSL}}{3 \sigma}=\frac{-0.007-(-0.015)}{3.0 .005}=0.520
\end{aligned}
$$

c. Cpk index

$\mathrm{Cpk}=\min \left(\frac{\mathrm{USL}-\mu}{3 \sigma}, \frac{\mu-\mathrm{LSL}}{3 \sigma}\right)$

$=\operatorname{Min}(\mathrm{Cpu}, \mathrm{Cpl})$

$=\min (0.485,0.520)$

$=0.485$

Cpk $\leq 1$, process uncapable

Whereas if Minitab software are as follows :

Standar Devi

a. Process Capability Ratio or Capability Process Index / Cp $\mathrm{Cp}=\frac{\mathrm{USL}-\mathrm{LSL}}{6 \sigma}$

$=\underline{0-(-0.015)}$

$$
\text { 6. } 0,014
$$

$=0.182$

So $\mathrm{Cp}<1$, Process uncapable

b. Upper and lower capability index

$$
\begin{aligned}
& \mathrm{CPU}=\frac{\mathrm{USL}-\mu}{3 \sigma}=\frac{0-(-0.009)}{3 \cdot 0.014}=0.218 \\
& \mathrm{CPL}=\frac{\mu-\mathrm{LSL}}{3 \sigma}=\frac{-0.009-(-0.015)}{3 \cdot 0.014}=0.145
\end{aligned}
$$

c. Cpk index

$$
\begin{aligned}
\mathrm{Cpk} & =\operatorname{Min}\left(\frac{\mathrm{USL}-\mu}{3 \sigma}, \frac{\mu-\mathrm{LSL}}{3 \sigma}\right) \\
=\operatorname{Min} & (\mathrm{Cpu}, \mathrm{Cpl}) \\
& =\operatorname{Min}(0.218,0.145) \\
& =0.145
\end{aligned}
$$

Cpk $\leq 1$, process uncapable

Whereas if Minitab software are as follows :

\section{Capability Process (CP) Connecting RodonMaret} $\mathbf{2 0 1 5}$ prior to the change as follows:

USL $=0$

LSL $=-0.015$

With an average of Data $=-0.007$

Standar Deviation $(\sigma)=0.014$ 


\section{International Journal of Science and Research (IJSR) \\ ISSN (Online): 2319-7064}

Index Copernicus Value (2013): 6.14 | Impact Factor (2015): 6.391

\begin{tabular}{|c|c|c|c|}
\hline \multirow{2}{*}{$\begin{array}{c}\text { Tanggal } \\
\text { Produksi } \\
\text { (diurutkan) }\end{array}$} & April 2015 & Mei 2015 & Juni 2015 \\
\hline & \multicolumn{3}{|c|}{$\begin{array}{c}\text { Diameter Hole } \\
\text { Standard } 666 \quad 0-0.015\end{array}$} \\
\hline $\mathbf{1}$ & -0.0060 & -00080 & -0.0100 \\
\hline 2 & -0.0080 & -000rr & -0.0120 \\
\hline 3 & -00000 & -00080 & -0.0150 \\
\hline 4 & $-0.0 \lcm{20}$ & -00162 & -0.0083 \\
\hline 5 & -0.0150 & -00095 & -0.0098 \\
\hline 6 & -0.0100 & -0000 & -0.0120 \\
\hline 7 & -0.0090 & $-000 r 8$ & -0.0110 \\
\hline $\mathbf{8}$ & -0.0140 & -00065 & -0.0091 \\
\hline 9 & -0.0110 & -00051 & $-0.00 \mathrm{rg}$ \\
\hline 10 & -0.0090 & -00080 & -0.0085 \\
\hline II & -0.0080 & -000 th & -0.0081 \\
\hline $\mathbf{L}$ & -0.0090 & -00005 & -0.0080 \\
\hline $\mathbf{B}$ & -0.0070 & -00085 & -0.0081 \\
\hline $\mathbf{1 4}$ & -0.0040 & -000:9 & -0.0081 \\
\hline $\mathbf{1 5}$ & -0.0075 & $-000=8$ & -0.00736 \\
\hline 16 & -0.0120 & -00036 & $-0.00 \%$ \\
\hline 13 & -0.0096 & -00090 & -0.0098 \\
\hline $\mathbf{~} \mathbf{8}$ & -0.0094 & -00092 & -0.0099 \\
\hline 乙9 & -0.0093 & $-000:-8$ & -0.0096 \\
\hline 20 & -0.0085 & -00005 & -00086 \\
\hline 21 & $-0.00 \mathrm{TH}$ & -00098 & -0.0084 \\
\hline
\end{tabular}
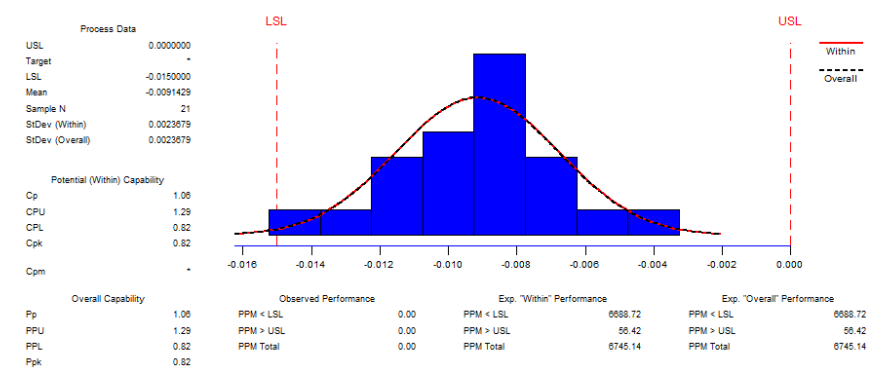

2. Capability Process (CP) Connecting Rodon May 2015prior to the change as follows:

USL $=0$

LSL $=-0.015$

With an average of Data $=-0.008$

Standar Deviation $(\sigma)=0.002$

a. Process Capability Ratio or Capability Process Index / Cp $\mathrm{Cp}=\underline{\mathrm{USL}}-\mathrm{LSL}$

$$
=\frac{0-(-0.015)}{6.0 .002}=1.337
$$

So $\mathrm{Cp}>1$, Proses baik (capable)

b. Index Kemampuan Proses Atas dan Kemampuan Proses Bawah (Upper and lower capability index)

1. Capability Process (CP) Connecting Rod onApril 2015prior to the change as follows:

$$
\begin{array}{ll}
\text { USL } & =0 \\
\text { LSL } & =-0.015
\end{array}
$$

With an average of Data $=-0.009$

Standar Deviation $(\sigma)=0.002$

$$
\begin{aligned}
& \mathrm{CPU}=\frac{\mathrm{USL}-\mu}{3 \sigma}=\frac{0-(-0.008)}{3 \cdot 0.002}=1.385 \\
& \mathrm{CPL}=\frac{\mu-\mathrm{LSL}}{3 \sigma}=\frac{-0.008-(-0.015)}{3.0 .002}=1.288 \\
& \text { c. } \mathrm{Cpk} \text { index } \\
& \mathrm{Cpk}=\min \left(\frac{\mathrm{USL}-\mu}{3 \sigma}, \frac{\mu-\mathrm{LSL})}{3 \sigma}\right. \\
& =\operatorname{Min}(\mathrm{Cpu}, \mathrm{Cpl}) \\
& =\min (1.385,1.288)(\mathrm{C} \\
& =1.288 \\
& \mathrm{Cpk} \geq 1, \text { process capable } \\
& \text { Whereas if Minitab software are as follows : }
\end{aligned}
$$

a. Process Capability Ratio or Capability Process Index / Cp

$$
\begin{aligned}
\mathrm{Cp} & =\frac{\mathrm{USL}-\mathrm{LSL}}{6 \sigma} \\
& =\frac{0-(-0.015)}{6.0,002} \\
& =1.069
\end{aligned}
$$
CP Diameter Hole Bulan Mei 2015

So $\mathrm{Cp}>1$, Process capable

b. Upper and lower capability index

$$
\begin{aligned}
& \mathrm{CPU}=\frac{\mathrm{USL}-\mu}{3 \sigma}=\frac{0-(-0.009)}{3 \cdot 0.002}=1.303 \\
& \mathrm{CPL}=\frac{\mu-\mathrm{LSL}}{3 \sigma}=\frac{-0.009-(-0.015)}{3.0 .002}=0.835
\end{aligned}
$$

c. Cpk index

$$
\begin{aligned}
\mathrm{Cpk}= & \min \left(\frac{\mathrm{USL}-\mu}{3 \sigma}, \frac{\mu-\mathrm{LSL}}{3 \sigma}\right) \\
= & \operatorname{Min}(\mathrm{Cpu}, \mathrm{Cpl}) \\
= & \min (1.303,0.835) \\
= & 0.835 \\
& \mathrm{Cpk} \leq 1, \text { process uncapable }
\end{aligned}
$$

Whereas if Minitab software are as follows :

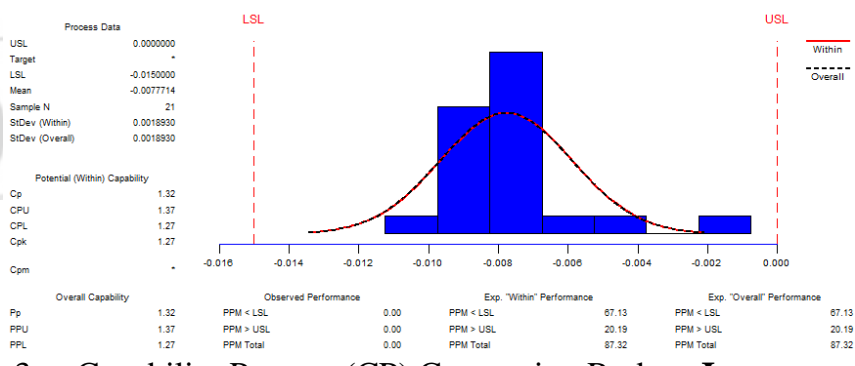

3. Capability Process (CP) Connecting Rod onJune 2015prior to the change as follows:

USL $=0$

LSL $=-0.015$

With an average of Data $=-0.009$

Standar Deviation $(\sigma) \quad=0.002$

a. Process Capability Ratio or Capability Process Index / Cp

$$
\begin{aligned}
\mathrm{Cp} & =\frac{\text { USL - LSL }}{6 \sigma} \\
& =\frac{0-(-0.015)}{6.0 .002} \\
& =1.364
\end{aligned}
$$

So $\mathrm{Cp}>1$, process capable 


\section{International Journal of Science and Research (IJSR) \\ ISSN (Online): 2319-7064}

Index Copernicus Value (2013): 6.14 | Impact Factor (2015): 6.391

b. Upper and lower capability index

$$
\begin{aligned}
& \mathrm{CPU}=\frac{\mathrm{USL}-\mu}{3 \sigma}=\frac{0-(-0.009)}{3 \cdot 0.002}=1.713 \\
& \mathrm{CPL}=\frac{\mu-\mathrm{LSL}}{3 \sigma}=\frac{-0.009-(-0.015)}{3 \cdot 0.002}=1.015
\end{aligned}
$$

c. Cpk index

$$
\mathrm{Cpk}=\min \left(\frac{\mathrm{USL}-\underline{\mu}}{3 \sigma}, \frac{\mu-\mathrm{LSL}}{3 \sigma}\right)
$$

$=\operatorname{Min}(\mathrm{Cpu}, \mathrm{Cpl})$

$=\min (1.713,1.015)$

$=1.015$

Cpk $\geq 1$, process capable

Whereas if Minitab software are as follows : CP Diameter Hole Bulan Juni 2015

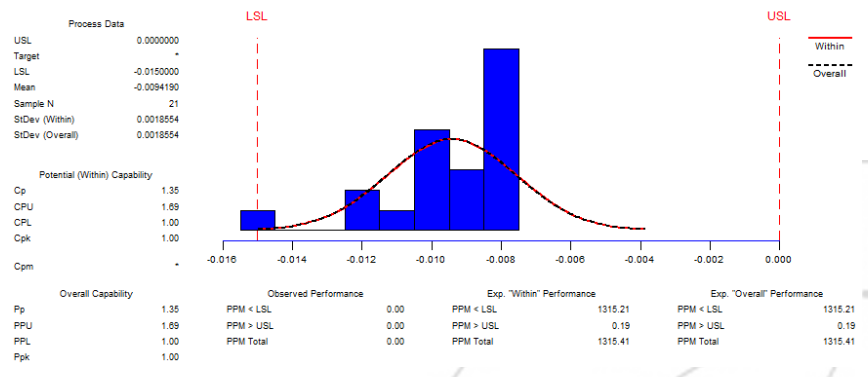

Cause dan Effect diagram

Cause and Effect diagram (fishbone diagram) is used to analyze the problem and the factors that cause the problem. Thus the diagram can be used to explain the causes of a problem.

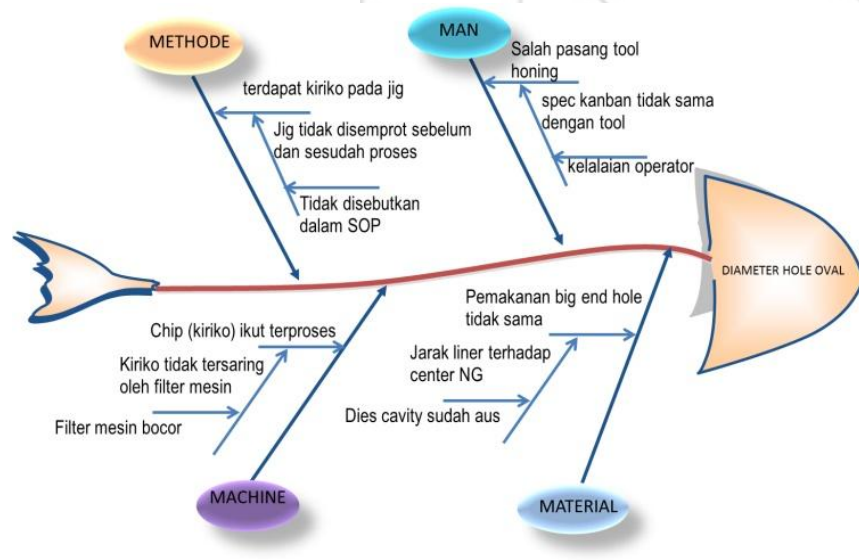

Figure 5: Fishbone Diagram

\section{Conclusion}

Based on the analysis and discussion, we conclude as follows:

a) Conditions diameter oval defect before repair reaches 7:47\% of the total production of approximately 26070 pcs or 1949 pcs. While the diameter oval defect condition after an improvement to $3.65 \%$ of the total production of 27053 pcs or about 988 pcs.

\begin{tabular}{|l|c|c|}
\hline & Jan - Maret 2015 & April - Juni 2015 \\
\hline Total Defect Produksi (pcs) & 1949 & 988 \\
\hline Total Produksi (pcs) & 26070 & 27053 \\
\hline \% Defect Produksi & $7.47 \%$ & $3.65 \%$ \\
\hline
\end{tabular}

b) Capability before the repair process from January 2015 - March 2015 respectively 0.1, 0:18, and 0.5, so it can be said that the process is not good. Meanwhile, after their process capability improvement in the month of April 2015 - June 2015 respectively 1.09,1.37,1.36 so that it can be said that a good process.

\begin{tabular}{|c|c|c|c|c|c|c|}
\hline \multirow{2}{*}{ ITEM } & \multicolumn{2}{|c|}{ SEBELUM PERBAIKAN } & \multicolumn{2}{c|}{ SETELAH PERBAIKAN } \\
\cline { 2 - 7 } & Jan'15 & Feb'15 & Mar'15 & Apr'15 & Mei'15 & Juni'15 \\
\hline CP & 0.10 & 0.18 & 0.50 & 1.09 & 1.37 & 1.36 \\
\hline $\mathrm{CPU}$ & 0.23 & 0.22 & 0.58 & 1.29 & 1.37 & 1.69 \\
\hline $\mathrm{CPL}$ & -0.03 & 0.14 & 0.53 & 0.82 & 1.27 & 1.00 \\
\hline $\mathrm{CPK}$ & -0.03 & 0.140 & 0.53 & 0.82 & 1.27 & 1.00 \\
\hline
\end{tabular}

\section{Suggestion}

Need to do continuous improvement (continuous improvement) that better quality performance in the future.

\section{References}

[1] Douglas C. Montgomery, Introduction to STATISTICAL QUALITY CONTROL 6th Edition, Arizona State University John Wiley \& Sons, Inc., by RR Donnelly (Jefferson city). 2009

[2] Dorothea Revelation Ariani, Quality Management, University of Atma Jaya Yogyakarta, June 1999

[3] Feigenbaum, Armand V., Total Qualit Control, Third Edition, McGraw-Hill, Inc. 1991

[4] D.C. Montgomery, Introduction to Statistical Process Control (Edition 3), Yogyakarta: Gadjah Mada University Press, 1995

[5] Marchal, et al. Techniques of Statistics in Business and Economics, Ketigabelas.Jakarta Edition: Four Salemba, 2007

[6] Dorothea Revelation Ariani, Statistical Quality Control: Qualitative Approach in Quality Management, University of Atma Jaya Yogyakarta, 2004

[7] Gryna, F. M .. Quality Planning and Analysis From Product Development Through Use (4th edition). Singapore: Mc-Graw Hill Int. Edition, 2001

[8] Antony, J. Ten Key Ingredients for Making SPC Succesful In Organisations Measuring Busines Excellence, 2000

[9] Caulcut, R. 1996. Responding To Changes.Quality and Reability Process Engineering International.

[10]Bird, D and Dale, B. The Misues and Abuse of SPC: A Case Study of the International Journal of Vehicle Design. 1994

[11]Xie, M. and Goh, T. N. Statistical Technique For Quality. The TQM Magazine, 1999

[12] Rungasamy, S; Antony, J; and Ghosh, S. 2002.Critical SPC Success Factors for Implementation In UK Small and Medium

[13] Enterprises: Some Key Finding From A Survey. The TQM Magazine.

[14] Garvin, David, Managing Quality. Inside Nasution, M. N., 2001, Integrated Quality Management

[15] Walpole, Ronald E. 1995. Introduction to Statistics Third Edition. Jakarta: PT. Gramedia Utama. 


\section{International Journal of Science and Research (IJSR) \\ ISSN (Online): 2319-7064}

Index Copernicus Value (2013): 6.14 | Impact Factor (2015): 6.391

[16] Iriawan, Nur. 2006. Statistics Processing data with Easy to Use Minitab 14. Yogyakarta: Andi Offset.

[17] Wignjosoebroto, Sritomo. 2006. Introduction to Industrial Engineering and Management. Surabaya: To Widya

[18] R. Sidartawan, ROTOR Journal, Volume 7 Number 2, November 2014

[19]Prof. Suharsimi Arikunto, Research Procedure A Practice Approach, (Cet.XII; Jakarta: Rineka Copyright, 2002)

[20]Hendra Poerwanto, "Plan-Do-Check-Act (PDCA), Quality Management, Web.19 June 2013

\section{Author Profile}

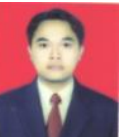

Nanang Supriana, received the ST. from Industrial Engineering STT. Wastukancana Purwakarta, West Java, Indonesia.

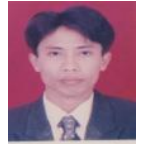

Muhammad Ali Akabar received the S.T., M.M.,Lecture of Industrial Engineering STT. Wastukancana Purwakarta, West Java, Indonesia. I had studied fromNational Institute of Technology Malang (2000), and Magister Management Strategicfrom Dipenogoro University (2003).

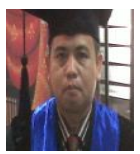

Osep Hijuzaman received the S.Pd., M.T, Lecture of STT. Wastukancana Purwakarta, West Java, Indonesia. I had studied in Electrical Engineering Educationfrom University of Indonesia in Bandung (1999) and Magister Industrial Engineering and Management from Pasundan University (2007), now studying at the University of Indonesia in Bandung, Doctoral Program in Management of Science. 